\title{
Diagnostic accuracy of CBNAAT (Gene-Xpert) Vs liquid culture in clinically diagnosed presumptive childhood tuberculosis admitted in Pt. J.N.M. Medical College \& Dr. Bhim Rao Ambedkar Memorial Hospital, Raipur (C.G.)
}

\author{
Phuljhele S. ${ }^{1}$, Beck P. ${ }^{2}$, Sinha A. ${ }^{3}$, Saroj A.K. ${ }^{4}$ \\ ${ }^{1}$ Dr. Sharja Phuljhele, Professor, ${ }^{2}$ Dr. Pratima Beck, Associate Professor, ${ }^{3}$ Dr. Ashish Sinha, Associate Professor, \\ ${ }^{4}$ Dr. Anil Kumar Saroj, Postgraduate fellow, all authors are attached with Department of Paediatrics, Pt. J. N. M. Medical \\ College, Raipur, C.G, India.
}

Corresponding Author: Dr. Pratima Beck, Department of Paediatrics, Pt. J. N. M. Medical College, Raipur, C.G., India. E-mail: anil.saroj4444@gmail.com,sharjaphuljhele@gmail.com,drpratima.beck@yahoo.com

\begin{abstract}
Background: Paediatric tuberculosis comprises $10 \%$ of all tuberculosis in developing countries. Smear microscopy is diagnostic for Pulmonary TB whereas for extra-pulmonary TB, CBNAAT (Gene-Xpert) and liquid-culture are available choices. Objectives: To establish the comparative diagnostic efficacy of CBNAAT and Liquid-culture in clinically presumptive childhood Tuberculosis. Outcome: Assess sensitivity and specificity of CBNAAT Vs Liquid-culture in clinically diagnosed presumptive childhood tuberculosis. Material \& Method: After approval from institutional ethical committee this Cross-sectional study was performed in department of paediatrics Pt. J. N. M. Medical College, Raipur C.G. Total 97 paediatric presumptive TB cases were enrolled for systematic screening from June-2017 to July-2018. Samples were collected and analysed by CBNAAT and liquid-culture. Data for Diagnostic efficacy of both techniques were analysed with appropriate statistical method. Results: Among study subject group 41.2\% belongs to 11-15 agegroup and $53.6 \%$ were female. Majority of them belongs to joint family (81.4\%) and low-socioeconomic (85.6\%) status. Among 97 presumptive cases CBNAAT was positive in $24.7 \%$ whereas liquid-culture was positive in $22.7 \%$. CBNNAT has sensitivity (100\%) and specificity (98.61\%) when compared with liquid-culture as gold-standard. It has $95.65 \%$ positive and $100 \%$ negative predictive values. CBNAAT had $100 \%$ of sensitivity and specificity in diagnosis of abdominal-tuberculosis and TB-meningitis. In Pulmonary-TB diagnosis its specificity reduced to $98 \%$. Conclusion: CBNAAT is equally efficacious diagnostic tool to diagnose clinically presumptive TB cases and it should be used in public health system for early diagnosis of paediatric tuberculosis.
\end{abstract}

Keywords: CBNAAT, Gene-Xpert, Tuberculosis.

\section{Introduction}

Tuberculosis (TB) is an infectious bacterial disease caused by Mycobacterium tuberculosis (M. TB). Worldwide, TB is a major health problem across the world. India is one of the high TB burden countries. As per the Global TB report 2017 the estimated incidence of TB in India was approximately $28,00,000$ accounting for about a quarter of the world's TB cases [1]. The actual burden of paediatric tuberculosis is not known, it is assumed that about $10 \%$ of total TB load is found in children. Globally one million cases of paediatric TB

Manuscript received: $10^{\text {th }}$ August 2019

Reviewed: $20^{\text {th }}$ August 2019

Author Corrected: $27^{\text {th }}$ August 2019

Accepted for Publication: $31^{\text {st }}$ August 2019 are estimated to occur every year, with more than 100,000 deaths. Childhood deaths from TB are usually caused by meningitis or disseminated disease. Mycobacterium tuberculosis remains to be one of the most significant causes of death from an infectious agent. Poor case ascertainment and limited surveillance data hamper the efforts to accurately quantify the disease burden associated with childhood TB [2].

Childhood TB is usually acquired from an infectious adult contact. High rates of transmission are sustained in TB-endemic areas due to high case density and prolonged diagnostic delay [3].As childhood TB reflects

Pediatric Review: International Journal of Pediatric Research Available online at: www.medresearch.in 467|P a g e 


\section{Original Research Article}

ongoing transmission, children are affected most acutely in areas where an adult $\mathrm{TB}$ epidemic is poorly controlled [4]. The global TB control strategy has focused predominantly on smear-positive cases and, therefore, not on childhood TB, which is usually paucibacillary and smear negative [3]. In addition, childhood TB remains neglected for various reasons, mainly the difficulty in diagnosing pulmonary $\mathrm{TB}$, the lack of scientific studies on childhood TB, the largely unknown outcomes of children with $\mathrm{TB}$, and the belief that childhood TB is not important for TB control $[3,5]$.

Xpert assay is not widely recognized as a diagnostic test for TB in India specifically in context of childhood tuberculosis. Further there is paucity of data on such diagnostic sensitivity and specificity of CB-NAAT (gene-Expert) in paediatric population in Chhattisgarh state. Currently WHO approved use of CBNAAT (MTB/RIF) diagnosing pulmonary and extra-pulmonary tuberculosis to simultaneously detect $M$. tuberculosis and rifampicin resistance mutations in the gene in 2011.CBNAAT can provide the results within 2 hours [6]. Xpert MTB/RIF assay is an automated CBNAAT.

In paediatric population many studies have demonstrated that Xpert assay is highly sensitive and specific in diagnosing for both pulmonary and extrapulmonary TB $[7,8,9,10]$. Hence this study is aimed to establish the diagnostic accuracy of clinically diagnosed presumptive childhood tuberculosis of CBNAAT versus liquid culture.

\section{Material and Methods}

Study setting: This study was conducted in Pt. JNM Medical college and Dr. Bhim Rao Ambedkar Memorial Hospital, Raipur (Pediatric WARDS \& PICU).

Type of study: Hospital based cross-sectional, observational study.

Study period: Data collection was done from August 2017 to July 2018 at Dr. Bhim Rao Ambedkar Memorial Hospital, Raipur in Paediatric department.

Sample size: Facility based time bound study so all children above mentioned study duration will included in the study. Total 97 children were enrolled for this study.

Method of sample collection, procedure and transport: Under all aseptic precaution, samples Pleural fluid, cerebrospinal fluid, gastric aspirate, ascetic fluid, pus aspirate from lymph node or cold abscess) were collected as per standard protocol in sterile container and sent for CBNAAT and liquid culture analysis.

The sample was divided equally into two parts, one part to be used for the CBNAAT and second for liquid culture (MGIT).

Complete blood count, Erythrocyte sedimentation rate, PPD, AFB, Chest x-ray and Ultrasonography and CT scan investigation were done to aid diagnosis along with clinical diagnosed presumptive tuberculosis.

Inclusion criteria: All suspected case of tuberculosis cases were included in this study after screening by various clinical and conventional methods including pleural effusion, cold abscess or lymphadenopathy, ascites and sinusitis, lymph node TB, Cold Abscess, TBM and Spinal TB, Genital TB, TB Skin.

Exclusion criteria: Confirmed case of pulmonary TB, pyogenic meningitis, non-tubercular effusion (cardiogenic, traumatic hypo-proteinaemia, cirrhosis, renal collagen vascular disorder and malignancy), malignant and inflammatory lymphadenopathy.

Ethical issues: approval taken from institutional ethical committee prior to study.

Data collection tools \& techniques: Data collection is divided in to three parts i.e. Interview schedule, sampling of specimen and clinical examination.

Data entry was done in Excel and analysis was done using SPSS 20.0 Wherever, possible percentage, Chisquare tests were applied.

Outcome and variables: Sensitivity, Specificity, Positive predictive value (PPV and Negative predictive value (NPV).

\section{Results}

Socio-demographic profile of cases: Distribution of age group in the 97 presumptive study subjects were 40 (41.2\%) 11-15 years of age followed by 31 (32.0\%) below 5 years, 20 (20.6\%) 6-10 years old while, $6(6.2 \%)$ more than 15 years age. Out of 97 subjects 52 (53.6\%) females and 45 (46.4\%) were males. All were immunized against BCG. Out of 97 presumptive cases $79(81.4 \%)$ were living in joint family and $18(18.6 \%)$ from nuclear family. Socio- economic status of cases shows that maximum, $83(85.6 \%)$ subjects belonged to low socio-economic status.

Pediatric Review: International Journal of Pediatric Research Available online at: www.medresearch.in 468|P a g e 


\section{Original Research Article}

History of tuberculosis contact \& BCG immunization status in the study subjects: It was seen that out of 97 cases 68 $(70.1 \%)$ cases had history of contact with tuberculosis. Immunization status in the study subjects was studied. It was found that all the subjects were immunised.

Observation of biochemical parameters: Table 1-showing mean PPD was found to be 12.65 \pm 2.35 . Mean ESR was observed to $33.82 \pm 23.84$. 29 presumptive cases had PPD more than $10 \mathrm{~mm}$ which is suggestive of TB infection and 41 study subjects had ESR more than $25 \mathrm{~mm} /$ hour. In subjects, minimum and maximum WBC was found to be $1100 / \mathrm{cumm}$ and 18000/cumm respectively with mean $9260 \pm 5100$. Mean Hb was noted to be $12.09 \pm 2.31 \mathrm{gm} \%$.

Table-1: Observation of biochemical parameters.

\begin{tabular}{|l|c|c|c|c|c|c|}
\hline Particulars & N & Min & Max & Mean & S.E. & S.D. \\
\hline ESR (mm/Hr) & 97 & 5 & 80 & 33.82 & 2.42 & 23.84 \\
\hline WBC (/cumm) & 97 & 1100 & 18000 & 9260 & 517.83 & 5100 \\
\hline HB (gm\%) & 97 & 5.6 & 16.2 & 12.09 & 0.23 & 2.31 \\
\hline PLT (10^3/cumm) & 97 & 125 & 310 & 167.37 & 2.92 & 28.73 \\
\hline
\end{tabular}

Type of fluid compared with AFB, CBNNAT and Liquid Culture results: Table 2 -showing Out of 97 samples different body fluids 10 were AFB positive, 22 CBNNAT and 21 Liquid cultures positive (Table 2) shows the type of fluid and positive results of samples by different diagnostic techniques.

Table-2: Type of fluid compared with AFB, CBNNAT and Liquid Culture results.

\begin{tabular}{|l|c|c|c|}
\hline \multirow{2}{*}{ Type of fluid } & AFB & CBNAAT & Liquid Culture \\
\cline { 2 - 4 } & Positive & Positive & Positive \\
\hline Sputum (16) & 1 & 7 & 7 \\
\hline Ascitic Fluid (2) & 1 & 1 & 1 \\
\hline Pleural Fluid (26) & 3 & 5 & 5 \\
\hline Gastric Aspirate (25) & 3 & 4 & 3 \\
\hline CSF (23) & 2 & 5 & 5 \\
\hline Total (97) & $\mathbf{1 0}$ & $\mathbf{2 2}$ & $\mathbf{2 1}$ \\
\hline
\end{tabular}

Chest X- ray, CT scan and Ultrasonography observations: The subjects were studied for their X- ray chest observations. It was found that maximum, i.e. 47 (48.5\%) subjects showed no abnormality. This was followed by pleural effusion (25.8\%) and consolidation (13.40\%). CT head findings in the study subjects were assessed.

CT was done in 15 cases out of that $9(60 \%)$ subjects had normal CT scan results. While 2 (13.3\%) subjects were found to have basal exudates, communicating hydrocephalus. ultrasound of chest and abdomen were done in 49 (50.5\%) subjects. $26(26.8 \%)$ subjects showed effusion. No abnormality was seen in $19(19.6 \%)$ subjects while, consolidation and ascitis were seen in $2(2.1 \%)$ and $1(1 \%)$ case respectively.

Clinical Features in presumptive study subjects: All the study subjects had low grade fever. (45) $46.39 \%$ were having difficulty in breathing, (17) $17.53 \%$ with cough more than 2 weeks and (20) $20.62 \%$ having meningeal sign, (16) $16.49 \%$ weight loss and (7) $7.22 \%$ had lymphadenopathy.

Comparison of clinically presumptive cases with CBNAAT \& Liquid Culture: (Table 3) Out of 69 Pulmonary TB presumptive cases, 18 were CBNAAT Positive and 17 positives in liquid culture $(\mathrm{P}=0.083)$. In 24 Tuberculosis meningitis cases, 5 were CBNAAT Positive and 4 positives in liquid culture $(\mathrm{P}=0.000)$. In 4 Abdominal tuberculosis cases; 1 CBNAAT positive and 1 liquid culture was positive. It was also statistically significant with CBNAAT $(\mathrm{P}=0.000)$. 
Table-3: Comparison of clinically presumptive cases with CBNAAT \& Liquid Culture.

\begin{tabular}{|l|c|c|c|c|}
\hline clinically presumptive cases & Frequency & CBNAAT Positive & $\begin{array}{c}\text { Liquid Culture } \\
\text { Positive }\end{array}$ & $\begin{array}{c}\text { P value } \\
\text { (CBNAAT) }\end{array}$ \\
\hline Pulmonary TB & 69 & 18 & 17 & 0.083 \\
\hline Tuberculous Meningitis & 24 & 5 & 4 & 0.000 \\
\hline Abdominal tuberculosis & 4 & 1 & 1 & 0.000 \\
\hline Total & 97 & 24 & 22 & \\
\hline
\end{tabular}

Diagnostic significance of CBNAAT compared to liquid culture: CBNAAT has sensitivity 100\%, specificity $98.61 \%$, Positive predicted value $95.65 \%$ and Negative predictive value is $100 \%$ as compare to liquid culture. (Graph: 2).

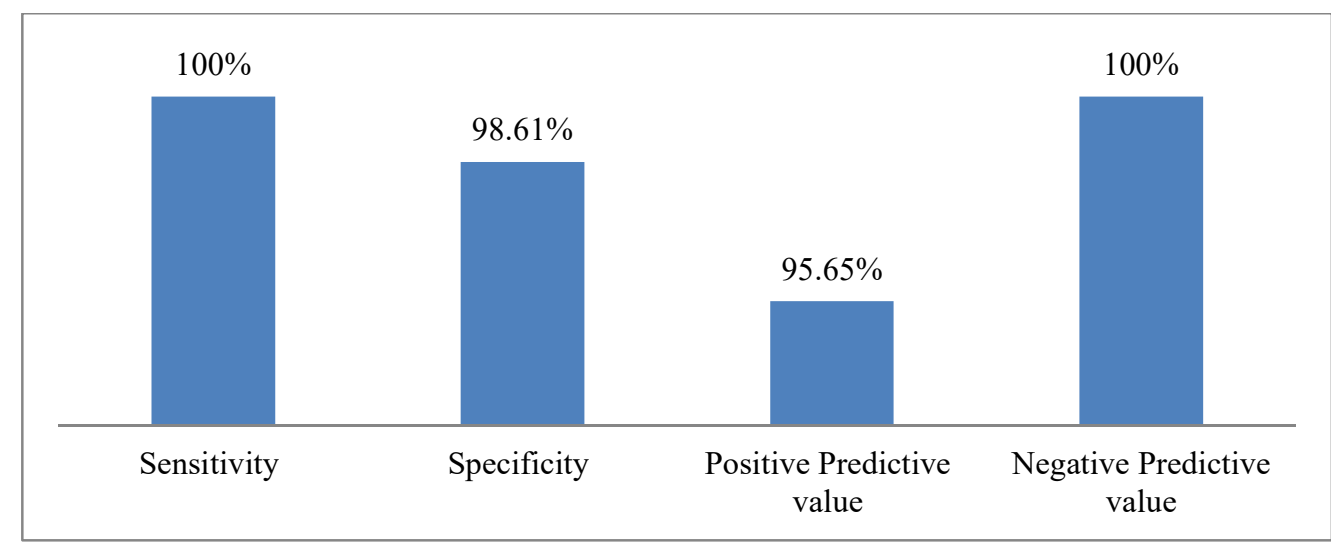

Figure 1: Diagnostic significance of CBNAAT compared to liquid culture

Diagnostic significance of CBNAAT in various types of Tuberculosis compared to Liquid culture: Abdominal and Tubercular meningitis has $100 \%$ sensitivity and specificity (Table 4).

Table-4: Diagnostic significance of CBNAAT in various types of Tb compared to Liquid culture

\begin{tabular}{|l|c|c|c|c|c|}
\hline Type of TB & Sensitivity\% & Specificity\% & PPV\% & NPV\% & p Value \\
\hline Abdominal TB & 100 & 100 & 100 & 100 & 0.083 \\
\hline Pulmonary TB & 100 & 98 & 93.8 & 100 & $<0.0001$ \\
\hline TB meningitis & 100 & 100 & 100 & 100 & $<0.0001$ \\
\hline
\end{tabular}

\section{Discussion}

In the Comparison of age and sex of study participants, $40(41.2 \%)$ subjects were of $11-15$ years of age. This was followed by $31(32.0 \%)$ subjects who were $</=5$ years of age. $20(20.6 \%)$ subjects were $6-10$ years old. out of 23 CBNAAT positive cases 16 were in age group of 10-16 years and 15 females were positive against 7 male children. Study by Sekadde et al (2013) in Uganda evaluated the performance of the Xpert MTB/RIF test for the diagnosis of childhood pulmonary tuberculosis using one induced sputum sample and described clinical characteristics. After excluding data from 15 subjects, the Xpert MTB/RIF test had a sensitivity of $79.4 \%$ and a specificity of $96.5 \%$. The Xpert MTB/RIF identified twice as many cases as the smear microscopy $(79.4 \%$
Vs $41.2 \%$ ). Age $>5$ years a history of Tuberculosis (TB) contact, $p$ value 0.03 , and a positive tuberculin skin test was associated with a positive Xpert MTB/RIF test. [11]. Similar results were found in our study in comparison to this study.

In present study BCG coverage of study participants was observed for scar mark. Out of 23 CBNAAT positive cases 19 were enrolled as students in schools and all of them had BCG immunization. According to various study BCG vaccine has documented protective efficacy against TB meningitis and miliary disseminated disease in children $(86 \%$ on average). It does not prevent primary infection and, more 


\section{Original Research Article}

importantly, does not prevent reactivation of latent pulmonary infection, the principal source of bacillary spread in the community. The impact of BCG vaccination on transmission of $\mathrm{M}$. tuberculosis is therefore limited. Hesseling AC, et al (2007) Western Cape Province, South Africa, stated that BCG vaccine has documented protective efficacy against TB meningitis and miliary disseminated disease in children ( $86 \%$ on average). It does not prevent primary infection and, more importantly, does not prevent reactivation of latent pulmonary infection, the principal source of bacillary spread in the community.

The impact of BCG vaccination on transmission of $\mathrm{M}$. tuberculosis is therefore limited $[12,13]$. In our study, we found that children with BCG immunization had also developed TB meningitis, which was similar to the study by Hesseling which also documented that only $86 \%$ of TB meningitis developed protection following immunization.

Out of 23 CBNNAT positive cases in our study, 18 were having active contact history. Cases of TB patients are a high-risk group for developing TB, particularly within the first year and children $<5$ years of age. A study by Fox GJ at el (2012 WHO consulted groupfound that Contacts of TB patients are a high-risk group for developing TB, particularly within the first year and children $<5$ years of age. Policy recommendations must consider evidence of the cost-effectiveness of various contact tracing strategies, and also incorporate complementary strategies to enhance case finding [14]. In our study, the history of contact with TB cases were higher in children $<5$ years of age which was similar to other studies.

In present study Biochemical parameters PPD and ESR were observed in presumptive cases. In the study subjects, mean PPD was found to be $167.37 \pm 28.73$ $10^{\wedge} 3 /$ cumm and $12.65 \pm 2.35$ respectively. Mean ESR was observed to $33.82 \pm 23.84$. Gandra et al (2017) Appolo Hyderabad study, Mean ESR was $112.09 \mathrm{~mm} /$ 1st $\mathrm{Hr} \pm 56.05$ (range $54-750 \mathrm{~mm} / 1 \mathrm{st} \mathrm{Hr}$ ). Mantoux test was positive in $270(75.42 \%)$. Positive GeneXpert assay (106 samples, 27.39\%; sputum $(87,24.23 \% \%$ ), pus (01), CSF (03), LNA $(15,57.69 \%)$ was reported in 87 patients [15]. The above study also had similar results in comparison to our study.

Comparison of suggestive X-ray findings and clinical feature with CBNAAT: out of total 97 presumptive suspected cases 47 had suggestive X-ray findings and out of that only 14 were CBNAAT positive. Giang et al
(2015) China noted X-ray findings consistent with tuberculosis in $95.3 \%$ cases. Thirty eight patients $(25.3 \%, \mathrm{n}=38 / 150)$ were classified as 'confirmed TB', 60 patients $(40.0 \%, \mathrm{n}=60 / 150)$ were 'probable TB' cases, $33(22.0 \% \mathrm{n}=33 / 150)$ patients were 'possible TB' cases, 17 patients were 'TB unlikely' (11.3\%, $\mathrm{n}=17 / 150)$ and 2 patients $(1.3 \%, \mathrm{n}=2 / 150)$ were classified as 'Not TB' with an alternative confirmed diagnosis. A study by Wekesa $\mathrm{C}$ at el (2014) found while 51 cases had CXR suggestive of TB $(\mathrm{P}=0.0018)$, of whom only 15 were Xpert-positive. CXR was suggestive of pulmonary TB in $15(71.4 \%)$ of the 21 patients with a positive Xpert test.

The majority of the sputum smear-negative patients did not have TB on single Xpert testing. CXR gave an overestimate of sputum smear-negative TB cases.[16]. Xray findings were less conclusive of TB in our study in comparison to the study held by Giang et al, but similar results were found in the study conducted by Wekesa $\mathrm{C}$ at el.

In present study various fluids were also compared with CBNAAT and Liquid culture testing. Type of fluid compared with AFB, CBNNAT and Liquid Culture shows that out of 24 CBNAAT cases 7 were sputum sample and 1 was AFB positive. Among 97 cases 25 were gastric aspirate sample out of that 4 CBNAAT and 3 Liquid cultures positive. And in same 4 gastric aspirate samples both CBNAAT and liquid culture were positive and in 1 no growth seen.

In rest of samples like Sputum, Ascitic fluid, pleural fluid and CSF, same number of CBNAAT positive and samples has seen culture growth. Diagnostic value of Gene-Xpert is significantly high as compared to ZiehlNeelsen smear microscopy and a useful tool in early diagnosis of tuberculosis [17].

The overall sensitivity, specificity, PPV and NPV of Gene-Xpert were $86.8 \%, 93.1 \%, 78.5 \%$ and $96 \%$ respectively. Gene-Xpert has a higher sensitivity than AFB smear microscopy in respiratory samples [18]. The overall positive rate of Xpert among sputum samples was significantly higher than that of liquid culture [19].

Both the studies show similar results of increased sensitivity of CBNAAT in comparison to AFB \& Liquid culture, probably due to the minimal amount of sample required for CBNAAT.

Also respiratory samples for CBNAAT are more sensitive compared to other samples. 


\section{Original Research Article}

In present study it was found that out of 97 subjects, 24 (24.7\%) subjects showed positive CBNAAT. In liquid culture observations $71(73.2 \%)$ had no growth, 22 $(22.7 \%)$ growth was seen and 4 (4.1\%) had contamination in sample.

Diagnostic significance of CBNAAT compared to liquid culture was Sensitivity (100\%), Specificity (98.61\%), Positive predictive value $(95.65 \%)$ and Negative Predictive value (100\%). In a similar study the overall sensitivity, specificity, PPV and NPV of GeneXpert were $86.8 \%, 93.1 \%, 78.5 \%$ and $96 \%$ respectively.

Gene-Xpert has a higher sensitivity than AFB smear microscopy in respiratory samples. Positive GeneXpert, but culture negative results should be read cautiously and be well correlated with clinical and treatment history of the patient [20]. In another study Gene Xpert MTB/RIF showed sensitivity, specificity and accuracy of $98.15 \%, 75 \%$ and $96.55 \%$ respectively in detection of mycobacterium tuberculosis. Gene Xpert MTB/RIF is a sensitive, specific and accurate test for both diagnosis of retreatment MTB and rifampicin resistance [21].

Limitation: First, one of the important strengths of the Xpert assay is its ability to detect the presence of Rifampicin resistance. The sensitivity and specificity of MTB/RIF assay to detect Rifampicin resistance in our study was not evaluated and not included in our objective as we didn't get the requisition for Rifampicin sensitivity by phenotypic method in all the positive samples. Second, as number of samples present in this study is less, further studies with a greater number of samples need to be done.

\section{Conclusion}

In this study CBNAAT shows significant association in diagnosis with other diagnostic modality i.e. liquid culture for pulmonary and extra-pulmonary TB.

Gene expert has overall sensitivity of $100 \%$ and specificity of $98.6 \%$. CBNAAT has highest diagnostic significance $(100 \%$ sensitivity and specificity) in tubercular meningitis as compare to pulmonary tuberculosis with $100 \%$ sensitivity and $98 \%$ specificity. From present study it is concluded that in highly clinically presumptive TB cases.

\section{What this adds to existing knowledge?}

The present study will provide vital inputs on applicability of gene expert as diagnostic test used for early diagnosis of paediatric age tuberculosis and help us to allocate role of CBNAAT as a screening tool, confirmatory test or supplementary test.

The yield of CBNAAT is $23.7 \%$. Thus, clinical evaluation cannot be excluded to treat tuberculosis patients in poor health care settings where CBNNAT and liquid culture is not available.

\section{Author contributions}

Dr. Sharja Phuljhele: Conceptualized, designed and analyzed the study.

Dr. Pratima Beck: Manuscript writing.

Dr. Ashish Sinha: Statistical analysis.

Dr. Anil Kumar Saroj: Conducted data-collection and help in analysis and manuscript writing.

Funding: Nil, Conflict of interest: None initiated, Permission from IRB: Yes

\section{References}

1. Gengenbacher M, Kaufmann SH. Mycobacterium tuberculosis: success through dormancy. FEMS Microbiol Rev. 2012; 36(3): 514-532. doi: 10.1111/j. 1574-6976.2012.00331.x. Epub 2012 Mar 8.

2. World Health Organization. (2018). Global tuberculosis report 2018. World Health Organization. https://apps.who.int/iris/handle/10665/274453. License: CC BY-NC-SA 3.0 IGO [Google scholar]

3. Swaminathan S, Rekha B. Pediatric tuberculosis: global overview and challenges. Clin Infect Dis. 2010 May 15;50(3):S184-S194. doi: 10.1086/651490.

4. Newton SM, Brent AJ, Anderson S, Whittaker E, Kampmann B. Paediatric tuberculosis. Lancet Infect Dis. 2008; 8(8):498-510. doi: 10.1016/S1473-3099(08) $70182-8$

5. Marais BJ. Childhood intra-thoracic tuberculosis: addressing the diagnostic dilemma (Doctoral dissertation, Stellenbosch: University of Stellenbosch).

6. Ben M. Childhood Tuberculosis: Epidemiology and Natural History of Disease. Indian J Pediat. 2011;78 (3): 321-327. doi: 10.1007/s12098-010-0353-1.

7. Nelson LJ, Wells CD. Global epidemiology of childhood tuberculosis. Int J Tuberc Lung Dis. 2004; 8 (5) : 636-647.

Pediatric Review: International Journal of Pediatric Research Available online at: www.medresearch.in 472|P a g e 


\section{Original Research Article}

8. Hsueh PR, Chen WH, Luh KT. Relationships between antimicrobial use and antimicrobial resistance in Gram-negative bacteria causing nosocomial infections from 1991-2003 at a university hospital in Taiwan. Int J Antimicrob Agents. 2005;26(6):463-472. doi: 10.1016/j.ijantimicag.2005.08.016.

9. Keflie TS, Ameni G. Microscopic examination and smear negative pulmonary tuberculosis in Ethiopia. Pan Afr Med J. 2014;19:162. doi: 10.11604/pamj. 2014. 19. 162. 3658 .

10.Siddiqi K, Lambert ML, Walley J. Clinical diagnosis of smear-negative pulmonary tuberculosis in lowincome countries: the current evidence. Lancet Infect Dis. 2003;3(5):288-296. doi: https://doi.org/ 10. 1016/ S1473-3099(03)00609-1

11. Boccia D, Hargreaves J, De Stavola BL, Fielding K, Schaap A,Godfrey-Faussett P, Ayles H. The association between household socioeconomic position and prevalent tuberculosis in Zambia: a case-control study. PLoS One. 2011;6(6): e20824. doi: 10.1371/ journal. pone. 0020824. Epub 2011 Jun 17.

12.Hesseling AC, Marais BJ, Gie RP, Schaaf HS, Fine PE, Godfrey-Faussett P, et al. The risk of disseminated Bacille Calmette-Guerin (BCG) disease in HIV-infected children. Vaccine. 2007; 25(1): 14-18. doi:10.1016/j. vaccine. 2006.07.020.

13. Fox GJ, Nhung NV, Sy DN, Britton WJ, Marks GB. Household contact investigation for tuberculosis in Vietnam: study protocol for a cluster randomized controlled trial. Trials. 2013;14:342. doi: 10.1186/17456215-14-342.

14. Munir MK, Rehman S, Aasim M, Iqbal R, Saeed S. Comparison of Ziehl Neelsen microscopy with GeneXpert for detection of Mycobacterium tuberculosis. IOSR J Dent Med Sci. 2015;14(11):56-60.

15. Rufai SB, Singh S, Singh A, Kumar P, Singh J, Vishal A. Performance of Xpert MTB/RIF on ascitic fluid samples for detection of abdominal tuberculosis. J Lab Phys. 2017;9(1):47-52. doi: 10.4103/0974-2727. 187927.

16. Pereira GR, Barbosa MS, Dias NJD, Almeida CPB, Silva DR. Impact of introduction of Xpert MTB/RIF test on tuberculosis (TB) diagnosis in a city with high TB incidence in Brazil. PLoS One. 2018;13(3): e0193988. doi: 10.1371/journal. pone. 0193988. E Collection 2018.

17. Agrawal M, Bajaj A, Bhatia V, Dutt S. Comparative Study of GeneXpert with ZN Stain and Culture in Samples of Suspected Pulmonary Tuberculosis. J Clin Diagn Res. 2016;10(5): DC09-12. doi: 10.7860/ JCDR/ 2016/ 18837.7755.

18. Shi J, Dong W, Ma Y, Liang Q, Shang Y, Wang F, Huang H, Pang Y. GeneXpert MTB/RIF Outperforms Mycobacterial Culture in Detecting Mycobacterium tuberculosis from Salivary Sputum. BioMed research international.2018;2018.doi: https://doi.org/10. 1155/ $2018 / 1514381$

19. Alsowey AM, Amin MI, Said AM. The Predictive Value of Multidetector High Resolution Computed Tomography in Evaluation of Suspected Sputum Smear Negative Active Pulmonary Tuberculosis in Egyptian Zagazig University Hospital Patients. Pol J Radiol. 2017; 82: 808-816. doi: 10.12659/PJR.903743. e Collection 2017.

20. van Zyl-Smit RN, Binder A, Meldau R, Mishra H, Semple PL, Theron G, Comparison of quantitative techniques including Xpert MTB/RIF to evaluate mycobacterial burden. PLoS One. 2011; 6(12): e28815. doi: 10.1371 journal.pone.0028815.

21. Khalil KF, Butt T. Diagnostic yield of Bronchoalveolar Lavage gene Xpert in smear-negative and sputum-scarce pulmonary tuberculosis. J Coll Physicians Surg Pak. 2015;25(2):115-118. doi: 02.2015/ JCPSP. 115118.

\section{How to cite this article?}

Phuljhele S, Beck P, Sinha A, Saroj A.K. Diagnostic accuracy of CBNAAT (Gene-Xpert) Vs liquid culture in clinically diagnosed presumptive childhood tuberculosis admitted in Pt. J.N.M. Medical College \& Dr. Bhim Rao Ambedkar Memorial Hospital, Raipur (C.G.). Int J Pediatr Res.2019;6(09):467-473.doi:10.17511/ijpr.2019.i09.05 